\title{
Checklist dos peixes marinhos do Estado de São Paulo, Brasil
}

\author{
Naércio Aquino Menezes ${ }^{1,2}$ \\ ${ }^{1}$ Museu de Zoologia, Universidade de São Paulo - USP, \\ Av. Nazaré, n. 481, CEP 04263-000, São Paulo, SP, Brasil \\ ${ }^{2}$ Autor para correspondência: Naércio Aquino Menezes, e-mail: naercio@usp.br
}

MENEZES, N.A. Checklist of marine fishes from São Paulo State, Brazil. Biota Neotrop. 11(1a): http://www. biotaneotropica.org.br/v11n1a/en/abstract?inventory+bn0031101a2011.

\begin{abstract}
The number of marine fish species listed for the coast of the São Paulo state is reasonably high in comparison with other Brazilian coastal regions of similar extension and mostly represented by demersal species that resulted from collecting efforts along the continental shelf. Species from rocky bottoms and reef areas as well as those from the deep sea are less abundant. Since the inventory is far from complete and no projects related to marine fishes have been carried out through the BIOTA/FAPESP program, it is expected that research groups involved in systematics, biology and other aspects related to the study of marine fishes be stimulated to contribute to increase of the knowledge of the group.
\end{abstract}

Keywords: marine fishes, biodiversity of the State of São Paulo, BIOTA/FAPESP Program.

Number of species: In the world: 15,950, in Brazil: 1,297, estimated in São Paulo State: 594.

MENEZES, N.A. Checklist dos peixes marinhos do Estado de São Paulo, Brasil. Biota Neotrop. 11(1a): http:// www.biotaneotropica.org.br/v11n1a/pt/abstract?inventory+bn0031101a2011.

Resumo: O número de espécies de peixes marinhos listadas para a costa do estado de São Paulo é razoavelmente alto em comparação com outras áreas do Brasil com extensão semelhante, a maior parte representada por espécies demersais que resultaram de coletas realizadas ao longo da plataforma continental. Espécies de fundos rochosos e áreas de recifes e de regiões profundas do oceano são menos abundantes. Como o inventário ainda é incompleto e nenhum projeto foi realizado através do programa BIOTA/FAPESP, espera-se que grupos de pesquisa engajados no estudo de sistemática, biologia e outros aspectos relativos aos peixes marinhos sejam estimulados a contribuir para aumentar o conhecimento atual do grupo.

Palavras-chave: peixes marinhos, biota paulista, Programa BIOTA/FAPESP.

Número de espécies: No mundo: 15.950, no Brasil: 1.297, estimadas no Estado de São Paulo: 594. 


\section{Introdução}

O termo "peixes" aqui utilizado tem uma conotação meramente convencional, ou seja, não representa um grupo monofilético no sentido filogenético e inclui tanto feiticeiras ou peixes-bruxa da Classe Myxini, como peixes cartilaginosos da Classe Chondrichthyes e peixes ósseos da Classe Actinopterygii.

A área costeira do Estado de São Paulo não representa uma região biogeograficamente definida, pois não há limites nítidos que permitam caracterizar uma ictiofauna marinha exclusiva. Muitas, senão a maior parte das espécies de peixes marinhos que ocorrem no litoral de São Paulo, existem também ao norte e ao sul, dentro de uma região que foi denominada Província biogeográfica Argentina (Rossi-Wongtschowski et al. 2009). Algumas espécies extrapolam os limites desta província, ocorrendo em outras áreas do Atlântico ocidental e, principalmente as espécies pelágicas, podem ter distribuição circunglobal. Por esta razão, é difícil calcular com certa precisão o número de espécies de peixes marinhos do litoral de São Paulo, mesmo com o auxílio de dados contidos na literatura mais recente. Embora muito melhor conhecida que a ictiofauna de água doce, como mencionado por Castro \& Menezes (1998), ainda existem lacunas de conhecimento em áreas carentes de inventariação como, por exemplo, os costões rochosos e áreas mais profundas do oceano. A maior parte dos esforços de coleta concentrou-se nos peixes demersais encontrados junto ao substrato da plataforma continental, através de vários projetos realizados por pesquisadores e estudantes do Instituto Oceanográfico da USP a partir de 1970 (Rossi-Wongtschowski et al., 2009). Mais recentemente, graças às atividades desenvolvidas durante o projeto "Conhecimento, conservação e utilização racional da diversidade da fauna de peixes do Brasil" - PRONEX, apoiado pelo CNPq (Menezes et al., 2003), os estudos de Moura (2003) contribuíram para o conhecimento da diversidade e riqueza dos peixes recifais. Por outro lado, também houve uma contribuição decisiva para o conhecimento da diversidade dos peixes pelágicos e batipelágicos através do Programa "Recursos Vivos da Zona Econômica Exclusiva - REVIZEE (Rossi-Wongtschowski et al. 2009).

\section{Métodos}

A lista das espécies apresentada nas Tabelas 1 e 2 foi obtida do "Catálogo das espécies de peixes marinhos do Brasil" (Menezes et al. 2003), que contém a relação mais atualizada das espécies que ocorrem

Tabela 1. Peixes marinhos das classes Myxini (1 e 2) e Chondrichthyes (3 a 107) do Estado de São Paulo.

Table 1. Marine fishes of the classes Myxini (1 and 2) and Chondrichthyes (3 to 107) of the São Paulo State.

\begin{tabular}{|c|c|c|c|}
\hline & Ordem & Família & Espécie \\
\hline 1 & Myxiniformes & Myxinidae & Eptatretus menezesi Mincarone, 2000 \\
\hline 2 & & & Myxine sotoi Mincarone, 2001 \\
\hline 3 & Carcharhiniformes & Carcharhinidae & Carcharhinus acronotus (Poey, 1860) \\
\hline 4 & & & Carcharhinus altimus (Springer, 1950) \\
\hline 5 & & & Carcharhinus brachyurus (Günther, 1870) \\
\hline 6 & & & Carcharhinus brevipinna (Müller \& Henle, 1839) \\
\hline 7 & & & Carcharhinus falciformis (Müller \& Henle, 1839) \\
\hline 8 & & & Carcharhinus isodon (Müller \& Henle, 1839) \\
\hline 9 & & & Carcharhinus leucas (Müller \& Henle, 1839) \\
\hline 10 & & & Carcharhinus limbatus (Müller \& Henle, 1839) \\
\hline 11 & & & Carcharhinus longimanus (Poey, 1861) \\
\hline 12 & & & Carcharhinus obscurus (Lesueur, 1818) \\
\hline 13 & & & Carcharhinus perezi (Poey, 1876) \\
\hline 14 & & & Carcharhinus plumbeus (Nardo, 1827) \\
\hline 15 & & & Carcharhinus porosus (Ranzani, 1839) \\
\hline 16 & & & Carcharhinus signatus (Poey, 1868) \\
\hline 17 & & & Galeocerdo cuvieri (Péron \& Lesueur, 1822) \\
\hline 18 & & & Negaprion brevirostris (Poey, 1868) \\
\hline 19 & & & Prionace glauca (Linnaeus, 1768) \\
\hline 20 & & & Rhizoprionodon lalandii (Müller \& Henle, 1839) \\
\hline 21 & & & Rhizoprionodon porosus (Poey, 1861) \\
\hline 22 & & Pseudotriakidae & Pseudotriakis microdon Capello, 1867 \\
\hline 23 & & Scyliorhinidae & Scyliorhinus haeckelii (Ribeiro, 1907) \\
\hline 24 & & Sphyrnidae & Sphyrna lewini (Griffith \& Smith, 1834) \\
\hline 25 & & & Sphyrna media Springer, 1940 \\
\hline 26 & & & Sphyrna mokarran (Ruppel, 1837) \\
\hline 27 & & & Sphyrna tiburo (Linnaeus, 1758) \\
\hline 28 & & & Sphyrna tudes (Valenciennes, 1822) \\
\hline 29 & & & Sphyrna zygaena (Linnaeus, 1758) \\
\hline 30 & & Triakidae & Galeorhinus galeus (Linnaeus, 1758) \\
\hline 31 & & & Mustelus canis (Mitchill, 1815) \\
\hline 32 & & & Mustelus higmani Springer \& Lowe, 1963 \\
\hline 33 & & & Mustelus schimtti Springer, 1939 \\
\hline
\end{tabular}


Tabela 1. Continuação...

\begin{tabular}{|c|c|c|c|}
\hline & Ordem & Família & Espécie \\
\hline 34 & Chimaeriformes & Callorhinchidae & Callorhichus callorhinchus (Linnaeus, 1758) \\
\hline 35 & & Rhinochimaeridae & Harriota raleighana Goode \& Bean, 1895 \\
\hline 36 & Hexanchiformes & Hexanchidae & Heptranchias perlo (Bonaterre, 1788) \\
\hline 37 & & Hexanchidae & Hexanchus griseus (Bonaterre, 1788) \\
\hline 38 & & Notorynchidae & Notorynchus cepedianus (Péron, 1807) \\
\hline 39 & Lamniformes & Alopiidae & Alopias superciliosus (lowe, 1841) \\
\hline 40 & & & Alopias vulpinus (Bonaterre, 1788) \\
\hline 41 & & Cetorhinidae & Cetorhinus maximus (Gunnerus, 1765) \\
\hline 42 & & Lamnidae & Carcharodon carcharias (Linnaeus, 1758) \\
\hline 43 & & & Isurus oxyrinchus Rafinesque, 1810 \\
\hline 44 & & & Isurus paucus Guitart Manday, 1966 \\
\hline 45 & & & Lamna nasus (Bonaterre, 1788) \\
\hline 46 & & Mitsukurinidae & Mitsukurina owstoni Jordan, 1898 \\
\hline 47 & & Odontaspididae & Carcharias taurus Rafinesque, 1810 \\
\hline 48 & & & Odontaspis ferox (Risso, 1810) \\
\hline 49 & & Odontaspididae & Odontaspis noronhai (Maul, 1955) \\
\hline 50 & & Pseudocarchariidae & Pseudocarcharias kamoharai (Matsubara, 1936) \\
\hline 51 & Orectolobiformes & Ginglymostomatidae & Ginglymostoma cirratum (Bonaterre, 1788) \\
\hline 52 & & Rhincodontidae & Rhyncodon typus Smith, 1828 \\
\hline 53 & Rajiformes & Dasyatidae & Dasyatis americana Hildebrand \& Schroeder, 1928 \\
\hline 54 & & & Dasyatis centroura (Mitchill, 1815 \\
\hline 55 & & & Dasyatis guttata (Bloch \& Schneider, 1801) \\
\hline 56 & & & Dasyatis sayi (Lesueur, 1817) \\
\hline 57 & & & Pteroplatytrygon violacea (Bonaparte, 1832) \\
\hline 58 & & Gymnuridae & Gymnura altavela Linnaeus, 1758) \\
\hline 59 & & & Gymnura micrura (Bloch \& Schneider, 1801) \\
\hline 60 & & Mobulidae & Manta birostris (Walbaum, 1792) \\
\hline 61 & & & Mobula hypostoma Bancroft, 1831) \\
\hline 62 & & & Mobula japanica (Müller\& Henle, 1841 \\
\hline 63 & & & Mobula tarapacana (Philippi, 1893) \\
\hline 64 & & & Mobula thurstoni (Lloyd, 1908) \\
\hline 65 & & Myliobatidae & Aetobatus narinari (Euphrasen, 1790) \\
\hline 66 & & & Myliobatis freminvillii Lesueur, 1824 \\
\hline 67 & & & Myliobatis goodei Garman, 1885 \\
\hline 68 & & & Rhinoptera bonasus (Mitchill, 1815) \\
\hline 69 & & & Rhinoptera brasiliensis (Müller, 1836) \\
\hline 71 & & Narcinidae & Benthobatis kreftti Rincón, Stehmann \&Vooren, 2001 \\
\hline 72 & & & Narcine brasiliensis (Olfers, 1831) \\
\hline 73 & & Pristidae & Pristis pectinata Latham, 1794 \\
\hline 74 & & & Pristis pristis (Linnaeus, 1758) \\
\hline 75 & & Rajidae & Atlantoraja castelnaui (Ribeiro, 1907) \\
\hline 76 & & & Atlantoraja cyclophora Regan, 1903) \\
\hline 77 & & & Bathyraja schroederi (Krefft, 1968) \\
\hline 78 & & & Dipturus menni Gomes \& Paragó, 2001 \\
\hline 79 & & & Gurgesiella dorsalifera McEachran \& Compagno, 1980 \\
\hline 80 & & & Psammobatis extenta (Garman, 1913) \\
\hline 81 & & & Psammobatis bergi Marini, 1932 \\
\hline 82 & & & Psammobatis lentiginosa McEachran, 1983 \\
\hline 83 & & & Psammobatis rutrum Jordan, 1891 \\
\hline 84 & & & Rajella sadowski (Krefft \& Stehmann, 1974) \\
\hline 85 & & & Rioraja agassizi (Müller \& Henle, 1841) \\
\hline 86 & & & Sympterygia acuta Garman, 1877 \\
\hline 88 & & Rhinobatidae & Rhinobatos horkelii Müller \& Henle, 1841 \\
\hline
\end{tabular}


Menezes, N.A.

Tabela 1. Continuação...

\begin{tabular}{|c|c|c|c|}
\hline & Ordem & Família & Espécie \\
\hline 89 & & & Rhinobatos percellens (Walbaum, 1792) \\
\hline 90 & & & Zapteryx brevirostris (Müller \& Henle, 1841) \\
\hline 91 & & Torpedinidae & Torpedo nobiliana Bonaparte, 1835 \\
\hline 92 & & & Torpedo puelcha Lahille, 1926 \\
\hline 93 & Squaliformes & Centrophoridae & Centrophorus granulosus (Bloch \& Schneider, 1801) \\
\hline 94 & & Dalatiidae & Dalatias licha (Bonaterre, 1788 \\
\hline 95 & & & Isistius brasiliensis (Quoy \& Gaimard) \\
\hline 96 & & & Squaliolus laticaudus Smith \& Radicliffe, 1912 \\
\hline 97 & & Echinorhinidae & Echinorhinus brucus (Bonaterre, 1788) \\
\hline 98 & & & Etmopterus bigelowi Shirai \& Tachikawa, 1993 \\
\hline 99 & & & Etmopterus lucifer Jordan \& Snyder, 1902 \\
\hline 100 & & Somniosidae & Centroscyminus coelolepis Bocage \& Capello, 1864 \\
\hline 101 & & & Centroscymnus cryptacanthus regan, 1906 \\
\hline 102 & & Squalidae & Cyrrhigaleus asper (Merret, 1973) \\
\hline 103 & & & Squalus acanthias Linnaeus, 1758 \\
\hline 104 & Squatiniformes & Squatinidae & Squatina argentina (Marini, 1930) \\
\hline 105 & & & Squatina dumeril Lesueur, 1818 \\
\hline 106 & & & Squatina guggenheim Marini, 1936 \\
\hline 107 & & & Squatina punctata Marini, 1936 \\
\hline
\end{tabular}

Tabela 2. Peixes marinhos da classe Actinopterygii do Estado de São Paulo.

Table 2. Marine fishes of the Class Actinopterygii of the São Paulo State.

\begin{tabular}{|c|c|c|c|}
\hline & Ordem & Família & Espécie \\
\hline 1 & Albuliformes & Albulidae & Albula vulpes (Linnaeus, 1758) \\
\hline 2 & & Notacanthidae & Notacanthus sexpinis Richardson, 1846 \\
\hline 3 & Anguilliformes & Chlopsidae & Chlopsis bicolor Rafinesque, 1810 \\
\hline 4 & & Congridae & Ariosoma opistophthalma (Ranzani, 1839) \\
\hline 5 & & & Bassanago albescens (Barnard, 1923) \\
\hline 6 & & & Conger orbignyanus Valenciennes, 1837 \\
\hline 7 & & & Rhechias dubius (Breder, 1927) \\
\hline 8 & & Muraenidae & Gymnothorax conspersus Poey, 1867 \\
\hline 9 & & & Gymnothorax funebris Ranzani, 1839 \\
\hline 10 & & & Gymnothorax moringa (Cuvier, 1829) \\
\hline 11 & & & Gymnothorax ocellatus Agassiz, 1831 \\
\hline 12 & & & Gymnothorax vicinus (Castelnau, 1855) \\
\hline 13 & & Nemichthyidae & Avocetinna acuticeps (Regan, 1916) \\
\hline 14 & & & Labichthys carinatus Gill \& Ryder, 1883 \\
\hline 15 & & Ophichthidae & Ahlia egmontis (Jordan, 1884) \\
\hline 16 & & & Myrichthys ocellatus (Lesueur, 1825) \\
\hline 17 & & & Ophichthus cylindroideus (Ranzani, 1840) \\
\hline 18 & & & Ophichthus gomesii (Castelnau, 1855) \\
\hline 19 & & & Ophicthus ophis (Linnaeus, 1758) \\
\hline 20 & & & Myrophis frio (Jordan \& Davis, 1891) \\
\hline 21 & & Synaphobranchidae & Dysommina rugosa Ginsburg, 1951 \\
\hline 22 & Atheriniformes & Atherinopsidae & Atherinella brasiliensis (Quoy \& Gaimard, 1825) \\
\hline 23 & & & Membras dissimilis (Carvalho, 1956) \\
\hline 24 & & & Odontesthes argentinensis (Valenciennes, 1835) \\
\hline 25 & & & Odontesthes iguapensis (Ribeiro, 1915) \\
\hline 26 & Aulopiformes & Alepisauridae & Alepisaurus brevirostris Gibbs, 1960 \\
\hline 27 & & & Alepisaurus ferox Lowe, 1833 \\
\hline 28 & & Chlorophthalmidae & Cholorophthalmus agassizi Bonaparte, 1840 \\
\hline
\end{tabular}


Tabela 2. Continuação...

\begin{tabular}{|c|c|c|c|}
\hline & Ordem & Família & Espécie \\
\hline 29 & & & Parasudis truculenta (Goode \& Bean, 1896) \\
\hline 30 & & Paralepididae & Sudis atrox Rofen, 1963 \\
\hline 31 & & & Sudis hyalina Rafinesque, 1810 \\
\hline 32 & & Synodontidae & Saurida brasiliensis Norman, 1935 \\
\hline 33 & & & Saurida caribbaea Breder, 1927 \\
\hline 34 & & & Synodus foetens (Linnaeus, 1766) \\
\hline 35 & & & Synodus synodus Linnaeus, 1758 \\
\hline 36 & & & Trachynocephalus myops (Forster, 1801) \\
\hline 37 & Batrachoidiformes & Batrachoididae & Thalassophryne montevidensis Berg, 1893 \\
\hline 38 & & & Thalassophryne nattereri Steindachner, 1876 \\
\hline 39 & & & Triathalossotia lambaloti Menezes \& Figueiredo, 1998 \\
\hline 40 & Beloniformes & Belonidae & Strongylura marina (Walbaum, 1792) \\
\hline 41 & & & Strongylura timиси (Walbaum, 1792) \\
\hline 42 & & Exocoetidae & Exocoetus obtusirostris Günther, 1866 \\
\hline 43 & & & Exocoetus volitans Linnaeus, 1758 \\
\hline 44 & & & Hirundichthys rondeletti (Valenciennes, 1847) \\
\hline 45 & & & Hirundichthys speculiger (Valenciennes, 1847) \\
\hline 46 & & Hemiramphidae & Hyporhamphus kronei Ribeiro, 1915 \\
\hline 47 & & & Hyporhamphus unifasciatus (Ranzani, 1841) \\
\hline 48 & Beryciformes & Anoplogastridae & Anoplogaster cornuta (Valenciennes, 1833) \\
\hline 49 & & Berycidae & Beryx splendens Lowe, 1834 \\
\hline 50 & & Holocentridae & Holocentrus adscensionis (Osbeck, 1765) \\
\hline 51 & & & Myripristis jacobus Cuvier, 1829 \\
\hline 52 & & & Plectrypops retrospinis (Guichenot, 1853) \\
\hline 53 & & & Sargocentron bullisi (Woods, 1955) \\
\hline 54 & & Trachychthyidae & Hoplostethus occidentalis Woods, 1973 \\
\hline 55 & Clupeiformes & Clupeidae & Brevoortia aurea Spix \& Agassiz, 1829 \\
\hline 56 & & & Brevoortia pectinata (Jenyns, 1842) \\
\hline 57 & & & Harengula clupeola (Cuvier, 1829) \\
\hline 58 & & & Harengula jaguana Poey, 1865 \\
\hline 59 & & & Opisthonema oglinum (Lesueur, 1818) \\
\hline 60 & & & Platanichthys platana (Regan, 1917) \\
\hline 61 & & & Sardinella aurita Valenciennes, 1847 \\
\hline 62 & & & Sardinella brasiliensis (Steindachner, 1879) \\
\hline 63 & & Engraulidae & Anchoa januaria Hildebrand, 1943 \\
\hline 64 & & & Anchoa marinii Hildebrand, 1943 \\
\hline 65 & & & Anchoviella lepidentostole (Fowler, 1941) \\
\hline 66 & & & Cetengraulis edentulus (Cuvier, 1829) \\
\hline 67 & & & Engraulis anchoita Hubbs \& Marini, 1935 \\
\hline 68 & & & Lycengraulis grossidens (Agassiz, 1829) \\
\hline 69 & & Pristigasteridae & Pellona harroweri (Fowler, 1919) \\
\hline 70 & Elopiformes & Magalopidae & Megalops atlanticus Valenciennes, 1846 \\
\hline 71 & Gadiformes & Macrouridae & Malacocephalus occidentalis Goode \& Bean, 1884 \\
\hline 72 & & Melanonidae & Melanonus zugmayeri Norman, 1929 \\
\hline 73 & & Merlucciidae & Merluccius hubsi Marini, 1932 \\
\hline 74 & & Moridae & Physiculus karrerae Paulin, 1988 \\
\hline 75 & & Phycidae & Urophycis brasiliensis (Kaup, 1858) \\
\hline 76 & & & Urophycis mystacea Ribeiro, 1903 \\
\hline 77 & Gasterosteiformes & Fistulariidae & Fistularia petimba Lacep`eede, 1803 \\
\hline 78 & & Macrorhamphosidae & Macrorhamphosus scolopax (Linnaeus, 1758) \\
\hline 79 & & & Notopogon fernandezianus (Delfin, 1899) \\
\hline
\end{tabular}


Menezes, N.A.

Tabela 2. Continuação...

\begin{tabular}{|c|c|c|c|}
\hline & Ordem & Família & Espécie \\
\hline 80 & & Syngnathidae & Cosmocampus elucens Poey, 1868) \\
\hline 81 & & & Hypocampus erectus Perry, 1810 \\
\hline 82 & & & Microphis brachyurus (Bleeker, 1853) \\
\hline 83 & & & Syngnathus folletti Herald, 1942 \\
\hline 84 & Lampridiformes & Lamprididae & Lampris guttatus (Brunnich, 1788) \\
\hline 85 & & Regalecidae & Regalecus glesne Ascanius, 1772 \\
\hline 86 & Lophiiformes & Antennariidae & Antennarius striatus (Shaw, 1794) \\
\hline 87 & & & Histrio histrio (Linnaeus, 1758) \\
\hline 88 & & Ceratiidae & Cryptosaras couesii Gill, 1883 \\
\hline 89 & & Lophiidae & Lophius gastrophysus Ribeiro, 1915 \\
\hline 90 & & Melanocetidae & Melanocetus johnsoni Günther 1864 \\
\hline 91 & & & Melanocetus murrayi, Günther, 1887 \\
\hline 92 & & Ogcocephalidae & Ogcocephalus vespertilio (Linnaeus, 1758 \\
\hline 93 & Mugiliformes & Mugilidae & Mugil curema Valenciennes, 1836 \\
\hline 94 & & & Mugil liza Valenciennes, 1836 \\
\hline 95 & & & Mugil platanus Günther, 1880 \\
\hline 96 & & & Mugil hospes Jordan \& Cuvier 1895 \\
\hline 97 & Myctophiformes & Myctophidae & Bolinichthys indicus (Nafpaktitis \& Nafpaktitis, 1969 \\
\hline 98 & & & Bolinichthys photothorax (Parr, 1928) \\
\hline 99 & & & Bolinichthys supralateralis (Parr, 1928) \\
\hline 100 & & & Centrobranchus nigroocellatus (Günther, 1873) \\
\hline 101 & & & Ceratoscopelus warmingii (Lütken, 1892) \\
\hline 102 & & & Diaphus anderseni Taning, 1932 \\
\hline 103 & & & Diaphus bertelseni Nafpaktitis, 1966 \\
\hline 104 & & & Diaphus brachycephalus Taning, 1928 \\
\hline 105 & & & Diaphus dumerilli (Bleeker,1856) \\
\hline 106 & & & Diaphus fragilis Taning, 1928 \\
\hline 107 & & & Diaphus metopoclamus (Cocco, 1829) \\
\hline 108 & & & Diaphus mollis Taning, 1928 \\
\hline 109 & & & Diaphus ostenfeldi Taning, 1932 \\
\hline 110 & & & Diaphus perspicillatus (Ogilby, 1898) \\
\hline 111 & & & Diaphus splendidus (Brauer, 1904 \\
\hline 112 & & & Gonichthys cocco (Cocco, 1829) \\
\hline 113 & & & Hygphum hygomii (Lütken, 1892) \\
\hline 114 & & & Hygophum reinhardti (Lütken, 1892) \\
\hline 115 & & & Hygophum taaning Bekker, 1965 \\
\hline 116 & & & Lampadena luminosa (Garman, 1899) \\
\hline 117 & & & Lampanyctus alatus Goode \& Bean, 1896 \\
\hline 118 & & & Lampanyctus australis Taning, 1932 \\
\hline 119 & & & Lampanyctus photonotos Parr, 1928 \\
\hline 120 & & & Lepidophanes gaussi (Brauer, 1906) \\
\hline 121 & & & Lepidophanes guentheri (Goode \& Bean, 1896) \\
\hline 122 & & & Lobianchia gemellari (Cocco, 1838) \\
\hline 123 & & & Myctophum affine (Lütken, 1892) \\
\hline 124 & & & Myctophum nitidulum Garman, 1899 \\
\hline 125 & & & Myctophum obtusirostre Taning, 1928 \\
\hline 126 & & & Nannobrachium cuprarium (Taning, 1928) \\
\hline 127 & & & Notolichnus valdiviae (Brauer, 1904) \\
\hline 128 & & & Notoscopelus caudispinosus (Johnson, 1863) \\
\hline 129 & & & Notoscopelus resplendens (Richardson, 1845) \\
\hline 130 & & & Symbolophorus rufinus Taning, 1928 \\
\hline
\end{tabular}


Tabela 2. Continuação...

\begin{tabular}{|c|c|c|c|}
\hline & Ordem & Família & Espécie \\
\hline 131 & Ophidiiformes & Carapidae & Echiodon dawsoni Williams \& Shipp, 1982 \\
\hline 132 & & Ophidiidae & Genipterus brasiliensis (Regan, 1903) \\
\hline 133 & & & Ophidion holbrooki Putnam, 1874 \\
\hline 134 & & & Raneya brasiliensis (Kaup, 1856) \\
\hline 135 & Osmeriformes & Argentinidae & Argentina striata Goode \& Bean, 1898 \\
\hline 136 & & & Glossanodon pygmaeus Cohen, 1958 \\
\hline 137 & & Opisthoproctidae & Winteria telescopa Brauer, 1901 \\
\hline 138 & Perciformes & Acanthuridae & Acanthurus bahianus Castelnau, 1855 \\
\hline 139 & & & Acanthurus chirurgus (Bloch, 1787) \\
\hline 140 & & & Acanthurus coeruleus Bloch \& Schneider, 1801 \\
\hline 141 & & & Acanthurus monroviae Steindachner, 1876 \\
\hline 142 & & Apogonidae & Apogon americanus Castelnau, 1855 \\
\hline 143 & & & Apogon planifrons Longley \& Hildebrand, 1940 \\
\hline 144 & & & Apogon pseudomaculatus Longley, 1932 \\
\hline 145 & & & Apogon quadrisquamatus Longley, 1934 \\
\hline 146 & & & Apogon puctitulatus (Poey, 1867) \\
\hline 147 & & Ariommatidae & Ariomma bondi Fowler, 1930 \\
\hline 148 & & Blenniidae & Hypleurochilus fissicornis Quoy \& Gaimard, 1824 \\
\hline 149 & & & Hypleurochilus psudoaequipinnis Bath, 1994 \\
\hline 150 & & & Hypsoblennius invemar Smith-Vaniz \& Acero,1980 \\
\hline 151 & & & Lupinoblennius paivai (Pinto,1958) \\
\hline 152 & & & Ophioblennius atlanticus (Valenciennes, 1836) \\
\hline 153 & & & Parablennius marmoreus (Poey, 1876) \\
\hline 154 & & & Parablennius pilicornis (Cuvier, 1829) \\
\hline 155 & & & Scartella cristata (Linnaeus, 1758) \\
\hline 156 & & Bramidae & Brama caribbea Mead, 1972 \\
\hline 157 & & & Brama dussumieri Cuvier, 1831 \\
\hline 158 & & & Pteraclis carolinus Valenciennes, 1833 \\
\hline 159 & & & Pterycombus petersii (Hilgendorf, 1878) \\
\hline 160 & & Callionymidae & Foetorepus dagmarae (Fricke, 1985) \\
\hline 161 & & Carangidae & Carangoides bartholomaei (Cuvier, 1833) \\
\hline 162 & & & Carangoides crysos (Mitchill, 1815) \\
\hline 163 & & & Carangoides ruber (Bloch, 1793) \\
\hline 164 & & & Caranx hippos (Linnaeus, 1758) \\
\hline 165 & & & Caranx latus Agassiz, 1831 \\
\hline 166 & & & Chloroscombrus chrysurus (Linnaeus, 1758) \\
\hline 167 & & & Decapterus punctatus (Cuvier, 1829) \\
\hline 168 & & & Hemicaranx amblyrhinchus (Cuvier, 1833) \\
\hline 169 & & & Naucrates ductor (Linnaeus, 1758) \\
\hline 170 & & & Oligoplites palometa (Cuvier, 1832) \\
\hline 171 & & & Oligoplites saliens (Bloch, 1793) \\
\hline 172 & & & Oligoplites saurus (Bloch \& Schneider, 1801) \\
\hline 173 & & & Parona signata (Jenyns, 1841) \\
\hline 174 & & & Pseudocaranx dentex (Bloch \& Schneider, 1801) \\
\hline 175 & & & Selene setapinnis (Linnaeus, 1758) \\
\hline 176 & & & Selene vomer (Linnaeus, 1758) \\
\hline 177 & & & Seriola dumerili (Risso, 1810) \\
\hline 178 & & & Seriola fasciata (Bloch, 1793) \\
\hline 179 & & & Seriola rivoliana Valenciennes, 1833 \\
\hline 180 & & & Seriola zonata (Mitchill, 1815) \\
\hline
\end{tabular}


Tabela 2. Continuação...

\begin{tabular}{|c|c|c|c|}
\hline & Ordem & Família & Espécie \\
\hline 181 & & & Trachinotus carolinus (Linnaeus, 1766) \\
\hline 182 & & & Trachinotus falcatus (Linnaeus, 1)766 \\
\hline 183 & & & Trachinotus goodei Jordan \& Evermann, 1896 \\
\hline 184 & & & Trachinotus marginatus Cuvier, 1832 \\
\hline 185 & & & Trachurus lathami Nichols, 1920 \\
\hline 186 & & & Uraspis secunda (Poey, 1860) \\
\hline 187 & & Centropomidae & Centropomus parallelus Poey, 1860 \\
\hline 188 & & & Centropomus undecimalis (Bloch, 1796 \\
\hline 189 & & Chaenopsidae & Emblemariopsis signifera (Ginsburg, 1942) \\
\hline 190 & & Chaetodontidae & Chaetodon sedentarius Poey, 1860 \\
\hline 191 & & & Chaetodon striatus Linnaeus, 1758 \\
\hline 192 & & & Prognathodes brasiliensis Burgess, 2001 \\
\hline 193 & & & Prognathodes guyanensis (Durand, 1960) \\
\hline 194 & & Cheilodactylidae & Nemadactylus bergi (Norman, 1937) \\
\hline 195 & & Clinidae & Ribeiroclinus eigenmanni (Jordan, 1888) \\
\hline 196 & & Coryphaenidae & Coryphaena equiselis Linnaeus, 1758 \\
\hline 197 & & & Coryphaena hippurus Linnaeus, 1758 \\
\hline 198 & & Dactyloscopidae & Dactyloscopus crossotus Starks, 1913 \\
\hline 199 & & & Dactyloscopus foraminosus Dawson, 1982 \\
\hline 200 & & & Dactyloscopus tridigitatus Gill, 1859 \\
\hline 201 & & Echeneidae & Echeneis naucrates Linnaeus, 1758 \\
\hline 202 & & & Phtheirichthys lineatus (Menzies, 1791) \\
\hline 203 & & & Remora australis (Bennett, 1840) \\
\hline 204 & & & Remora brachyptera (Lowe, 1839) \\
\hline 205 & & & Remora osteochir (Linnaeus, 1758) \\
\hline 206 & & & Remora remora (Linnaeus, 1758) \\
\hline 207 & & & Remorina albescens (Temminck \& Schlegel, 1845 \\
\hline 208 & & Eleotridae & Dormitator maculatus (Bloch, 1792) \\
\hline 209 & & & Eleotris pisonis (Gmelin, 1789) \\
\hline 210 & & & Guavina guavina (Valenciennes, 1837) \\
\hline 211 & & Ephippidae & Chaetodipterus faber (Broussonet, 1782) \\
\hline 212 & & Gempylidae & Gempylus serpens Cuvier, 1829 \\
\hline 213 & & & Lepidocybium flavobrunneum (Smith, 1843) \\
\hline 214 & & & Prometichthys prometeus (Cuvier, 1832) \\
\hline 215 & & & Ruvettus pretiosus Cocco, 1833 \\
\hline 216 & & & Thyrsitops lepidopoides (Cuvier, 1832) \\
\hline 217 & & Gerreidae & Diapterus auratus Ranzani, 1842 \\
\hline 218 & & & Diapterus rhombeus (Valenciennes, 1830) \\
\hline 219 & & & Eucinostomus argenteus Baird \& Girard, 1855 \\
\hline 220 & & & Eucinostomus gula (Quoy \& Gaimard, 1824) \\
\hline 221 & & & Eucinostomus melanopterus (Bleeker, 1863) \\
\hline 222 & & & Eugerres brasilianus (Valenciennes, 1830) \\
\hline 223 & & & Ulaema lefroyi (Goode, 1874) \\
\hline 224 & & Gobiidae & Awaos tajasica (Lichtenstein, 1822) \\
\hline 225 & & & Barbulifer ceuthoecus (Jordan \& Gilbert, 1884) \\
\hline 226 & & & Bathygobius soporator (Valenciennes, 1837) \\
\hline 227 & & & Chriolepis vespa Hasting \& Bortone, 1981 \\
\hline 228 & & & Coryphopterus glaucofrenum Gill, 1863 \\
\hline 229 & & & Ctenogobius boleosoma (Jordan \& Gilbert, 1882) \\
\hline 230 & & & Ctenogobius shufeldti (Jordan \& Eigenmann, 1887) \\
\hline
\end{tabular}


Tabela 2. Continuação...

\begin{tabular}{|c|c|c|}
\hline & Família & Espécie \\
\hline 231 & & Ctenogobius smaragdus (Valenciennes, 1837) \\
\hline 232 & & Ctenogobius stigmaticus (Poey, 1860) \\
\hline 233 & & Elacatinus figaro Sazima, Moura \& Rosa, 1997 \\
\hline 234 & & Evorthodus lyricus (Girard, 1858) \\
\hline 235 & & Gnatholepis thompsoni Jordan, 1904 \\
\hline 236 & & Gobioides broussonetii Lacepede, 1800 \\
\hline 237 & & Gobionellus oceanicus (Pallas, 1770) \\
\hline 238 & & $\begin{array}{l}\text { Gobiosoma hemigymnum (Eigenmann \& Eigenmann, } \\
\text { 1888) }\end{array}$ \\
\hline 239 & & Gobiosoma nudum Meek \& Hildebrand, 1928 \\
\hline 240 & & Lythripnus brasiliensis Greenfield, 1988 \\
\hline 241 & & Microgobius carri Fowler, 1945 \\
\hline 242 & & Microgobius meeki Evermann \& Marsh, 1899 \\
\hline 243 & & Parrela macropteyx Ginsburg, 1939 \\
\hline 244 & Grammatidae & Gramma brasiliensis Sazima, Gasparini \& Moura,1998 \\
\hline 245 & Haemulidae & Anisotremus surinamensis (Bloch, 1791) \\
\hline 246 & & Anisotremus virginicus (Linnaeus, 1758) \\
\hline 247 & & Boridia grossidens Cuvier, 1830 \\
\hline 248 & & Conodon nobilis (Linnaeus, 1758) \\
\hline 249 & & Genyatremus luteus (Bloch, 1790) \\
\hline 250 & & Haemulon aurolineatum Cuvier, 1830 \\
\hline 251 & & Haemulon parra (Desmarest, 1823) \\
\hline 252 & & Haemulon plumieri (Lacepede, 1801) \\
\hline 253 & & Haemulon steindachneri (Jordan \& Gilbert, 1882) \\
\hline 254 & & Orthopristis ruber (Cuvier, 1830) \\
\hline 255 & & Pomadasys crocro (Cuvier, 1830) \\
\hline 256 & Istiophoridae & Makaira nigricans Lacepede, 1802 \\
\hline 257 & & Tetrapturus albidus Poey, 1860 \\
\hline 258 & & Tetrapturus pfluegeri Robins \& de Sylva, 1963 \\
\hline 259 & Labridae & Bodianus pulchellus (Poey, 1860) \\
\hline 260 & & Bodianus rufus (Linnaeus, 1758) \\
\hline 261 & & Decodon puellaris (Poey, 1860) \\
\hline 262 & & Doratonotus megalepis Günther, 1862 \\
\hline 263 & & Halichoeres brasiliensis (Bloch, 1791) \\
\hline 264 & & Halichoeres poeyi (Steindachner, 1867) \\
\hline 265 & & Thalassoma noronhanum (Boulenger, 1890) \\
\hline 266 & & Xirichthys novacula (Linnaeus, 1758) \\
\hline 267 & Labrisomidae & Labrisomus cricota Sazima, Gasparini \& Moura, 2002 \\
\hline 268 & & Labrisomus kalisherae (Jordan, 1904) \\
\hline 269 & & Malacoctenus delalandii (Valenciennes, 1836) \\
\hline 270 & & Paraclinus rubicundus (Starks, 1913) \\
\hline 271 & & Paraclinus spectator Guimarães \& Bacellar, 2002 \\
\hline 272 & & Starksia brasiliensis (Gilbert, 1900) \\
\hline 273 & Lobotidae & Lobotes surinamensis (Bloch, 1790) \\
\hline 274 & Lutjanidae & Lutjanus alexandrei Moura \& Lindeman, 2007 \\
\hline 275 & & Pristipomoides freemani Anderson, 1966 \\
\hline 276 & Malacanthidae & Caulolatilus chrysops (Valenciennes, 1833) \\
\hline 277 & & Lopholatilus villari Ribeiro, 1915 \\
\hline 278 & & Malacanthus plumieri (Bloch, 1786) \\
\hline 279 & Mullidae & Mullus argentinae Hubbs \& Marini, 1933 \\
\hline 280 & & Psudupeneus maculatus (Bloch, 1793) \\
\hline
\end{tabular}


Tabela 2. Continuação...

\begin{tabular}{|c|c|c|}
\hline & Família & Espécie \\
\hline 281 & & Upeneus parvus Poey, 1852 \\
\hline 282 & Opistognathidae & Lonchopisthus meadi Menezes \& Figueiredo, 1971 \\
\hline 283 & & Opistognathus brasiliensis Smith-Vaniz, 1997 \\
\hline 284 & Percophidae & Bembrops heterurus (Ribeiro, 1903) \\
\hline 285 & & Percophis brasiliensis Quoy \& Gaimard, 1825 \\
\hline 286 & Pinguipedidae & Pinguipes brasilianus Cuvier, 1829 \\
\hline 287 & & Pseudopercis numida Ribeiro, 1903 \\
\hline 288 & & Pseudopercis semifasciata (Cuvier, 1829) \\
\hline 289 & Polynemidae & Polydactylus oligodon (Günther, 1860) \\
\hline 290 & & Polydactylus virginicus (Linnaeus, 1758) \\
\hline 291 & Pomacanthidae & Centropyge aurantonotus Burgess, 1974 \\
\hline 292 & & Holacanthus ciliaris (Linnaeus, 1758) \\
\hline 293 & & Holacanthus tricolor (Bloch, 1795) \\
\hline 294 & & Pomancanthus paru (Bloch, 1787) \\
\hline 295 & Pomacentridae & Abudefduf saxatilis (Linnaeus, 1758) \\
\hline 296 & & Chromis enchrysura Jordan \& Gilbert, 1882 \\
\hline 297 & & Chromis flavicauda (Günther, 1880) \\
\hline 298 & & Chromis jubauna Moura, 1995 \\
\hline 299 & & Chromis multilineata (Guichenot, 1853) \\
\hline 300 & & Stegastes fuscus (Cuvier, 1830) \\
\hline 301 & & Stegastes pictus (Castelnau, 1855) \\
\hline 302 & & Stegastes uenfi Novelli, Nunan \& Lima, 2000 \\
\hline 303 & & Stegastes variabilis (Castelnau, 1855) \\
\hline 304 & Pomatomidae & Pomatomus saltatrix (Linnaeus, 1766) \\
\hline 305 & Priacanthidae & Cookeolus japonicus (Cuvier, 1829) \\
\hline 306 & & Heteropriacanthus cruentatus (Lacepede, 1801) \\
\hline 307 & & Priacanthus arenatus Cuvier, 1829 \\
\hline 308 & Rachycentridae & Rachycentron canadum (Linnaeus, 1766) \\
\hline 309 & Scaridae & Cryptotomus roseus (Cope, 1871) \\
\hline 310 & & Nicholsina usta (Valenciennes, 1840) \\
\hline 311 & & Scarus guacamaia Cuvier, 1829 \\
\hline 312 & & Scarus trispinosus Valenciennes, 1840 \\
\hline 313 & & Scarus zelindae Moura, Figueiredo \& Sazima, 2001 \\
\hline 314 & & Sparisoma amplum (Ranzani, 1841) \\
\hline 315 & & Sparisoma axillare (Steidachner, 1878) \\
\hline 316 & & Sparisoma frondosum (Agassiz, 1831) \\
\hline 317 & & Sparisoma radians (Valencienes, 1840) \\
\hline 318 & Sciaenidae & Bairdiella ronchus (Cuvier, 1830) \\
\hline 319 & & Ctenosciaena gracilirrhus (Metzelaar, 1919) \\
\hline 320 & & Cynoscion acoupa (Lacepede, 1801) \\
\hline 321 & & Cynoscion guatucupa (Cuvier, 1830) \\
\hline 322 & & Cynoscion jamaicensis (Vaillant \& Bocourt, 1883) \\
\hline 323 & & Equetus lanceolatus (Linnaeus, 1758) \\
\hline 324 & & Isopisthus parvipinnis (Cuvier, 1830) \\
\hline 325 & & Larimus breviceps Cuvier, 1830 \\
\hline 326 & & Lonchurus lanceolatus (Bloch, 1788) \\
\hline 327 & & Macrodon ancylodon (Bloch \& Schneider, 1801) \\
\hline 328 & & Menticirrhus americanus (Linnaeus, 1758) \\
\hline 329 & & Menticirrhus littoralis (Holbrook, 1847) \\
\hline 330 & & Micropogonias furnieri (Desmarest, 1823) \\
\hline
\end{tabular}


Tabela 2. Continuação...

\begin{tabular}{|c|c|c|}
\hline & Família & Espécie \\
\hline 331 & & Odontoscion dentex (Cuvier, 1830) \\
\hline 332 & & Ophioscion punctatissimus Meek \& Hildebrand, 1925 \\
\hline 333 & & Paralonchurus brasiliensis (Steindachner, 1875) \\
\hline 334 & & Pareques umbrosus (Jordan \& Eigenmann, 1889) \\
\hline 335 & & Pogonias cromis (Linnaeus, 1766) \\
\hline 336 & & Stellifer brasiliensis (Schultz, 1945) \\
\hline 337 & & Stellifer rastrifer (Jordan, 1889) \\
\hline 338 & & Stellifer sp. \\
\hline 339 & & Umbrina canosai Berg, 1895 \\
\hline 340 & & Umbrina coroides Cuvier, 1830 \\
\hline 341 & Scombridae & Acanthocybium solandri (Cuvier, 1832) \\
\hline 342 & & Allothunnus fallai Servently, 1948 \\
\hline 343 & & Auxis rochei (Risso, 1810$)$ \\
\hline 344 & & Auxis thazard (Lacepede, 1800) \\
\hline 345 & & Euthynnus alleteratus (Rafinesque, 1810) \\
\hline 346 & & Katsuwonus pelamis (Linnaeus, 1758) \\
\hline 347 & & Sarda sarda (Bloch, 1793) \\
\hline 348 & & Scomber colias Gmelin, 1789 \\
\hline 349 & & $\begin{array}{l}\text { Scomberomorus brasiliensis Collette, Russo \& Zavala- } \\
\text { Camin, } 1978\end{array}$ \\
\hline 350 & & Scomberomorus cavalla (Cuvier, 1829) \\
\hline 351 & & Thunnus alalunga (Bonaterre, 1788) \\
\hline 352 & & Thunnus albacares (Bonaterre, 1788) \\
\hline 353 & & Thunnus atlanticus (Lesson, 1831) \\
\hline 354 & & Thunnus obesus (Lowe, 1839) \\
\hline 355 & & Thunnus thynnus (Linnaeus, 1758) \\
\hline 356 & Serranidae & Acanthistius brasilianus (Cuvier, 1828) \\
\hline 357 & & Acanthistius patachonicus (Jenyns, 1840) \\
\hline 358 & & Anthias menezesi Anderson \& Heemstra, 1980 \\
\hline 359 & & Bathyanthias roseus Günther, 1880 \\
\hline 360 & & Cephalopholis fulva (Linnaeus, 1758) \\
\hline 361 & & Diplectrum formosum (Linnaeus, 1766) \\
\hline 362 & & Diplectrum radiale (Quoy \& Gaimard, 1824) \\
\hline 363 & & Dules auriga Cuvier, 1829 \\
\hline 364 & & Epinephelus flavolimbatus Poey, 1865 \\
\hline 365 & & Epinephelus morio (Valenciennes, 1828) \\
\hline 366 & & Epinephelus niveatus (Valenciennes, 1828) \\
\hline 367 & & Hemanthias vivanus (Jordan \& Swain, 1885) \\
\hline 368 & & Mycteroperca acutirostris (Valenciennes, 1828) \\
\hline 369 & & Mycteroperca interstitialis (Poey, 1860) \\
\hline 370 & & Polyprion americanus (Bloch \& Schneider, 1801) \\
\hline 371 & & Pronotogrammus martinicensis (Guichenot, 1868) \\
\hline 372 & & Rypticus randalli Courtenay, 1967 \\
\hline 373 & & Serranus atrobranchus (Cuvier, 1829) \\
\hline 374 & & Serranus baldwini (Evermann \& Marsh, 1899) \\
\hline 375 & & Serranus flaviventris (Cuvier, 1829) \\
\hline 376 & & Serranus phoebe Poey, 1851 \\
\hline 377 & Sparidae & Archosargus aries (Valenciennes, 1830) \\
\hline 378 & & Archosargus rhomboidalis (Linnaeus, 1758) \\
\hline 379 & & Calamus penna (Valenciennes, 1830) \\
\hline 380 & & Calamus pennatula Guichenot, 1868 \\
\hline
\end{tabular}


Tabela 2. Continuação...

\begin{tabular}{|c|c|c|c|}
\hline & Ordem & Família & Espécie \\
\hline 381 & & & Diplodus argenteus (Valenciennes, 1830) \\
\hline 382 & & & Pagrus pagrus (Linnaeus, 1758) \\
\hline 383 & & Sphyraenidae & Sphyraena barracuda (Edwards, 1771) \\
\hline 384 & & & Sphyraena guachancho Cuvier, 1829 \\
\hline 385 & & & Sphyraena tome Fowler, 1903 \\
\hline 386 & & Stromateidae & Peprilus paru Linnaeus, 1758 \\
\hline 387 & & Trichiuridae & Evoxymetopon taeniatus Gill, 1863 \\
\hline 388 & & & Lepidopus altifrons Parin \& Collette, 1993 \\
\hline 389 & & & Trichiurus lepturus Linnaeus, 1758 \\
\hline 390 & & Tripteygiidae & Enneanectes altivelis Rosenblatt, 1960 \\
\hline 391 & & Uranoscopidae & Astroscopus sexspinosus (Steindachner, 1876) \\
\hline 392 & & & Astroscopus y-graecum (Cuvier, 1829) \\
\hline 393 & & Xiphiidae & Xiphias gladius Linnaeus, 1758 \\
\hline 394 & Pleuronectiformes & Achiridae & Achirus declivis Chabanaud, 1940 \\
\hline 395 & & & Achirus lineatus (Linnaeus, 1758) \\
\hline 396 & & & Cathataridium garmani (Jordan, 1889) \\
\hline 397 & & & Gymanchirus nudus Kaup, 1858 \\
\hline 398 & & & Trinectes paulistanus Ribeiro, 1915 \\
\hline 399 & & Bothidae & Bothus robinsi Topp \& Hoff, 1972 \\
\hline 400 & & & Monolene antillarum Norman, 1933 \\
\hline 401 & & Cynoglossidae & Symphurus diomedianus (Goode \& Bean, 1885) \\
\hline 402 & & & Symphurus ginsburgi Menezes \& Benvegnú, 1976 \\
\hline 403 & & & Symphurus jenynsi Evermann \& Kendall, 1906 \\
\hline 404 & & & Symphurus kyaropterygium Menezes \& Benvegnú, 1976 \\
\hline 405 & & & Symphurus tesselatus (Quoy \& Gaimard, 1824) \\
\hline 406 & & & Symphurus trewavasae Chabanaud, 1948 \\
\hline 407 & & Paralichthyidae & Citarichthys macrops Dresel, 1855 \\
\hline 408 & & & Citarichthys spilopterus Günther, 1862 \\
\hline 409 & & & Etropus crossotus Jordan \& Gilbert, 1882 \\
\hline 410 & & & Etropus longimanus Norman, 1933 \\
\hline 411 & & & Paralichthys orbignyanus (Valenciennes, 1839) \\
\hline 412 & & & Paralichthys patagonicus Jordan, 1889 \\
\hline 413 & & & Paralichthys triocellatus Ribeiro, 1903 \\
\hline 414 & & & Syacium papillosum (Linnaeus, 1758) \\
\hline 415 & & & Verecundum rasile Jordan, 1891 \\
\hline 416 & Polymixiiformes & Polymixiidae & Polymixia lowei Günther, 1859 \\
\hline 417 & Scorpaeniformes & Agonidae & Agonus cataphractus (Linnaeus, 1758) \\
\hline 418 & & Dactylopteridae & Dactylopterus volitans (Linnaeus, 1758) \\
\hline 419 & & Peristediidae & Peristedion altipinne Regan, 1903 \\
\hline 420 & & Scorpaenidae & Helicolenus lahillei Norman, 1937 \\
\hline 421 & & & Idiaston kyphos Eschemeyer, 1964 \\
\hline 422 & & & Phenascorpius nebris Eschemeyer, 1965 \\
\hline 423 & & & Pontinus corallinus Ribeiro, 1903 \\
\hline 424 & & & Scorpaena brasiliensis Cuvier, 1829 \\
\hline 425 & & & Scorpaena calcarata Goode \& Bean, 1882 \\
\hline 426 & & & Scorpaena grandicornis Cuvier, 1829 \\
\hline 427 & & & Scorpaena isthmensis Meek \& Hildebrand, 1928 \\
\hline 428 & & & Scorpaena plumieri Bloch, 1789 \\
\hline 429 & & & Scorpaenodes tredecimspinosus Metzelaar, 1919 \\
\hline 430 & & & Setarches guentheri Johnson, 1862 \\
\hline 431 & & Triglidae & Bellator brachychir (Regan, 1914) \\
\hline
\end{tabular}


Tabela 2. Continuação...

\begin{tabular}{|c|c|c|c|}
\hline & Ordem & Família & Espécie \\
\hline 432 & & & Prionotus nudigula Ginsburg, 1950 \\
\hline 433 & & & Prionotus punctatus (Bloch, 1793) \\
\hline 434 & Siluriformes & Ariidae & Bagre bagre (Linnaeus, 1758) \\
\hline 435 & & & Bagre marinus (Mitchill, 1815) \\
\hline 436 & & & Cathorops agassizi (Eigenmann \& Eigenmann, 1888) \\
\hline 437 & & & Genidens genidens (Cuvier, 1829) \\
\hline 438 & & & Genidens machadoi (Ribeiro, 1918) \\
\hline 439 & Stomiiformes & Sternoptychidae & Argyropelecus hemigymnus Cocco, 1829 \\
\hline 440 & & & Argyropelecus sladeni Regan, 1908 \\
\hline 441 & & & Maurolicus stehmanni Parin \& Kobyliansky, 1993 \\
\hline 442 & & & Sternoptyx diaphana Hermann, 1781 \\
\hline 443 & & & Sternoptyx pseudobscura Baird, 1971 \\
\hline 444 & & Stomiidae & Astronesthes macropogon Goodyear \& Gibbs, 1970 \\
\hline 445 & & & Chauliodus sloani (Schneider, 1801) \\
\hline 446 & & & Echiostoma barbatum Lowe, 1843 \\
\hline 447 & & & Eustomias filifer (Gilchrist, 1906) \\
\hline 448 & & & Eustomias spherulifer Gibbs, Clarke \& Gomon, 1983 \\
\hline 449 & & & Flagellostomias boureei (Zugmayer, 1913) \\
\hline 450 & & & Leptostomias gladiator (Zugmayer, 1911) \\
\hline 451 & & & Melanostomias macrophotus Regan \& Trewawas, 1930 \\
\hline 452 & & & Melanostomias melanops Brauer, 1902 \\
\hline 453 & & & Melanostomias niger Gilchrist \& Von Bonde, 1924 \\
\hline 454 & & & Melanostomias valdivie Brauer, 1902 \\
\hline 455 & & & Photonectes braueri (Zugmayer, 1913) \\
\hline 456 & & & Stomias affinis Günther, 1887 \\
\hline 457 & & & Stomias boa (Risso, 1810) \\
\hline 458 & Tetraodontiformes & Balistidae & Balistes capriscus Gmelin, 1789 \\
\hline 459 & & Diodontidae & Cyclichthys spinosus (Linnaeus, 1758) \\
\hline 460 & & & Diodon holocanthus Linnaeus, 1758 \\
\hline 461 & & & Diodon hystrix Linnaeus, 1758 \\
\hline 462 & & Molidae & Mola mola (Linnaeus, 1758) \\
\hline 463 & & & Ranzania laevis (Pennant, 1776) \\
\hline 464 & & Monacanthidae & Aluterus heudelotii Hollard, 1855 \\
\hline 465 & & & Aluterus monoceros (Walbaum, 1792) \\
\hline 466 & & & Aluterus schoepfi (Walbaum, 1792) \\
\hline 467 & & & Cantherhines macrocerus (Hollard, 1853) \\
\hline 468 & & & Cantherhines pullus (Ranzani, 1842) \\
\hline 469 & & & Monacanthus ciliatus (Mitchill, 1818) \\
\hline 470 & & & Stephanolepis hispidus (Linnaeus, 1766) \\
\hline 471 & & Ostraciidae & Acanthostracion polygonius Poey, 1876 \\
\hline 472 & & Ostraciidae & Acanthostracion quadricornis (Linnaeus, 1758) \\
\hline 473 & & Tetraodontidae & Canthigaster figueredoi Moura \& Castro, 2002 \\
\hline 474 & & & Lagocephalus laevigatus (Linnaeus, 1758) \\
\hline 475 & & & Sphoeroides greeleyi Gilbert, 1900 \\
\hline 476 & & & Sphoeroides pachygaster (Müller \& Troschel, 1848) \\
\hline 477 & & & Sphoeroides spengleri (Bloch, 1758) \\
\hline 478 & & & Sphoeroides testudineus (Linnaeus, 1758) \\
\hline 479 & & & Sphoeroides tyleri Shipp, 1972 \\
\hline 480 & Zeiformes & Caproidae & Antigonia capros Lowe, 1843 \\
\hline 481 & & Zeidae & Zenopsis conchifer (Lowe, 1852) \\
\hline
\end{tabular}


na costa brasileira, elaborada por um conjunto de especialistas nos diversos grupos de peixes representados. Neste catálogo, a ocorrência de cada espécie foi determinada através de pesquisa exaustiva da literatura, sendo incluídas apenas aquelas efetivamente documentadas através de descrições originais ou exame de material catalogado em coleções de museus.

O número estimado de espécies de peixes marinhos e estuarinos na região costeira que inclui o estado de São Paulo (594) é inferior a 650, número estimado em Rossi-Mongtschowski et al. (2009), mas o critério aqui adotado é distinto, uma vez que só foram incluídas as espécies explicitamente citadas como ocorrendo no litoral de São Paulo ou cuja distribuição conhecida inclui esta região. As espécies citadas no referido catálogo como distribuindo-se, por exemplo, no Atlântico ocidental até o leste do Brasil não foram consideradas.

\section{Resultados e Discussão}

Levando-se em conta a relativa uniformidade ambiental na região costeira do Estado de São Paulo, onde predomina uma ictiofauna marinha constituída por peixes demersais devido a existência de uma ampla plataforma continental com substrato de lama e areia (Rossi-Wongtschowski et al. 2009), a riqueza, considerando-se o número estimado de espécies é razoavelmente alta. Não há abundância de peixes associados à presença de fundos consolidados e recifes coralinos, como acontece em outras regiões do Nordeste do Brasil, mas quando comparada à ictiofauna de regiões mais ao sul, ainda mais pobres de tais formações, a diversidade no litoral de São Paulo é maior. A região costeira do Estado do Rio Grande do Sul, por exemplo, também caracterizada pela existência de ampla plataforma continental dentro da mesma Província Argentina e com extensão costeira equivalente à do Estado de São Paulo, tem cerca de 350 espécies de peixes marinhos e estuarinos, número estimado através do mesmo critério aqui indicado. A seu favor o estado do Rio Grande do Sul tem os cruzeiros realizados na plataforma continental de março de 1968 a abril de 1969 (GEDIP 1) e em 1977 (GEDIP 2), com a utilização do R/V "Prof. W. Besnard” do Instituto Oceanográfico da USP (vide descrição mais detalhada em Menezes et al. 1997). Os arrastos foram realizados entre 10 e 200 metros de profundidade, capturando-se um grande número de espécies demersais.

No Programa BIOTA/FAPESP não houve nenhum projeto relacionado a peixes marinhos o que, espera-se, venha a ocorrer proximamente. Como a inventariação da fauna de peixes marinhos e estuarinos ainda é incompleta, projetos visando documentar o que existe através de coletas em áreas menos conhecidas precisam ser elaborados nos próximos anos. Paralelamente, projetos de pesquisa dirigidos ao conhecimento da biologia (alimentação, reprodução, ciclo de vida, etc.) das espécies são fundamentais para proporcionar o conhecimento básico necessário, principalmente das espécies comercialmente exploradas, para orientar decisões objetivando a elaboração de planos de gestão e manejo da pesca. Como muitas das espécies atualmente pescadas estão sobrexplotadas (Rossi-Wontschowski et al. 2009), medidas urgentes, embasadas em dados confiáveis, precisam ser tomadas para garantir a sobrevivência destas espécies nos próximos anos.
Os principais grupos de pesquisa do estado de São Paulo engajados no estudo de peixes marinhos exercem suas atividades no Instituto Oceanográfico (IOUSP), Museu de Zoologia (MZUSP), ambos da Universidade de São Paulo e Instituto de Pesca - SAA/ APTA, sediado em Santos. No primeiro, os estudos são mais dirigidos a aspectos biológicos, enquanto no segundo a pesquisa é voltada mais à sistemática e biogeografia.

A maior parte dos exemplares resultantes dos projetos realizados por pesquisadores e alunos do IOUSP durante campanhas de curta e longa duração foram depositados, mediante acordo, na coleção ictiológica do MZUSP, que representa o maior acervo do país. $\mathrm{O}$ estudo deste material tem possibilitado o desenvolvimento de dissertações e teses. O Instituto de Pesca tem seus objetivos mais relacionados à pesca no estado de São Paulo.

Apesar dos esforços realizados para inventariar a fauna de peixes marinhos do estado, há lacunas decorrentes da inexistência de coletas em áreas pouco exploradas, como costões e fundos rochosos, utilizando aparelhos diversificados, como sugerido por Castro \& Menezes (1998).

\section{Agradecimentos}

Carmen Lúcia D.B. Rossi-Wongtschowski (IOUSP) e José Lima de Figueiredo (MZUSP) leram o manuscrito e acrescentaram valiosas observações.

\section{Referências Bibliográficas}

CASTRO, R.M.C. \& MENEZES, N.A. 1998. Estudo diagnóstico da diversidade de peixes do estado de São Paulo. In Biodiversidade do estado de São Paulo, Brasil: síntese do conhecimento ao final do século XX. Vertebrados (C.A. Joly \& C.E.M. Bicudo, orgs.). Programa BIOTA/ FAPESP, São Paulo, v.6, p.3-13.

MENEZES, N.A., FIGUEIREDO, J.L. \& BRITSKI, H.A. 1997. Ichthyological collection building at the Museu de Zoologia da Universidade de São Paulo. In Collection Building in Ichthyology and Herpetology. American Society of Ichthyologists and Herpetologists (T.W. Pietsch \& W.D. Anderson Jr., eds.). Special Publication Number 3, p.561-565.

MENEZES, N.A., BUCKUP, P.A, FIGUEIREDO, J.L. \& MOURA, R.L. (eds.) 2003. Catálogo das espécies de peixes marinhos do Brasil. São Paulo: Museu de Zoologia, 159 p.

MOURA, R.L. 2003. Riqueza de espécies, diversidade e organização de assembléias de peixes em ambientes recifais: um estudo ao longo do gradiente latitudinal da costa brasileira. Tese de doutorado, Instituto de Biociências da Universidade de São Paulo.

NELSON, J.S. 2006. Fishes of the world. 4th ed. John Wiley \& Sons, Hoboken, New Jersey, 601p.

ROSSI-WONGTSCHOWSKI, C.L.D.B., VAZ-DOS-SANTOS, A.M., RODRIGUES DA COSTA, M., FIGUEIREDO, J.L., ÁVILA-DASILVA, A.D., LEÃO DE MOURA, R. \& MENEZES, N.A. 2009. Peixes Marinhos. In Fauna ameaçada de extinção no Estado de São Paulo (P.M. Bressan, M.C.M. Kierulf \& A.M. Sugieda, coords.). Fundação Parque Zoológico de São Paulo; Secretaria do Meio Ambiente, São Paulo, p.427-567. 